\title{
Uso de internet en pacientes que acuden a consulta hospitalaria de urología
}

\author{
Santos Arrontes D, García González JI, Martín Muñoz MP, Jiménez Jiménez JI, Paniagua Andrés P.
}

Servicio de Urología. Hospital de Móstoles. Móstoles. Madrid.

Actas Urol Esp. 2007;31(10):1161-1165

\begin{abstract}
RESUMEN
USO DE INTERNET EN PACIENTES QUE ACUDEN A CONSULTA HOSPITALARIA DE UROLOGÍA

Hipótesis: El aumento en los conocimientos de informática de la población general y la difusión de Internet como herramienta de comunicación y conocimiento, permite a los pacientes tener un mayor entendimiento de sus patologias.

Objetivo: Evaluar el uso de Internet por parte de la población de un área sanitaria y el conocimiento extraído sobre sus patologias de carácter urológico.

Material y Métodos: En este estudio prospectivo se incluyeron todos los paciente mayores de edad que acudieron a consulta hospitalaria de urología entre el 1 de Septiembre y el 31 de Diciembre de 2006, en un área sanitaria de 200,000 habitantes. A todos los pacientes se les entregó un cuestionario autoadministrado para su cumplimentación, no interviniendo el facultativo en su desarrollo en ningún caso. Las variables a estudio fueron la edad (menores de 30 años, entre 30 y 60 años y mayores de 60 años), el sexo, patología del paciente (únicamente se valorarán aquellas con al menos 5 casos), el nivel educativo (ninguno, graduado escolar, estudios medios y estudios universitarios), presencia de ordenador en su casa (si/no), conocimiento de la existencia de internet ( $\mathrm{si} / \mathrm{no}$ ), búsquedas realizadas sobre patologias urológicas e influencia de dichas consultas en su relación con el médico. Se evaluará la relación entre el uso de internet y las distintas variables mediante el test de Kruskall-Wallis. Se considerará significativa una verosimilitud de la hipótesis nula inferior a 0,05 .

Resultados: En total se recibieron 1.111 cuestionarios de los cuales fueron útiles para su procesamiento 1062. La edad media fue de 60,98 , con un error estándar de 15,08. El 18,4\% fueron mujeres. La distribución poblacional por nivel de estudios fue: $22,2 \%$ sin estudios, $43,5 \%$ con graduado escolar, 27,5 con un nivel educativo medio y un $6,8 \%$ de universitarios. El 58,4 \% de los pacientes niega tener ordenador en casa, el $37,7 \%$ desconoce lo que es internet, el 76,7 \% carece de dirección de correo electrónico y únicamente el 6,7 \% visitan páginas médicas pero sólo el 1,5\% reconocen haber preguntado a su médico sobre la información recibida en internet. Según la categorización por edades, los pacientes con edades inferiores a 30 años tienen significativamente unos mayores conocimientos en informática e internet ( $\mathrm{p}<0,001)$. No obstante no se han evidenciado diferencias estadísticamente significativas entre la edad y el hecho de preguntar sobre la información recibida a través de internet $(\mathrm{p}=0,1)$. Las páginas web más visitadas fueron por orden de más a menos visitadas: tuotromedico.com, varicocele.com, aecc.es, wikipedia.com, prostatitis.org, ondasalud.com y mapfrecajasalud.com. Las patologías más buscadas fueron: prostatitis crónica ( $25 \%$ de los pacientes afectos), cáncer de testículo ( $20 \%$ de los pacientes), varicocele ( $18,7 \%$ ), enfermedad de Peyronie o incurvación congénita peneana (18,1\%) y estenosis de la unión pielo-uretereral (16,6\%).

Conclusiones: - La explotación de internet como herramienta de información por parte de los pacientes es muy baja, debido a las características inherentes de la población, como son el bajo nivel de estudios. - Las páginas web urológicas deberian dedicar una parte amplia para la patología más frecuente en los grupos de población más jóvenes, como el varicocele o la prostatitis. No obstante, es lógico esperar que estos patrones epidemiológios se modifiquen con el paso del tiempo. - Se deberia fomentar el uso de internet y de la informática en general entre los distintos grupos poblacionales en el área sanitaria a estudio.
\end{abstract}

Palabras clave: Internet. Cuestionario. Epidemiologia.

\section{ABSTRACT}

INTERNET USE IN PATIENTS ATTENDING A HOSPITAL UROLOGY CLINIC

Hypothesis: The increase in the awareness of computers in the general population and the spread of Internet as a tool for communication and knowledge, allows patients to have greater understanding of their conditions.

Objective: To evaluate the use of Internet by the population from a health area and the knowledge extracted about their urological conditions.

Material and Methods: This prospective study included all patients of age who attended a hospital urology clinic between 1st September and 31 st December 2006, in a health area of 200,000 inhabitants. All patients were given a self-administered questionnaire to complete; medical staff did not intervene in filling it out in any case. The study variables were age (under 30, between 30 and 60 and over 60 ), sex, patient's pathology (only those with at least 5 cases were assessed), educational level (none, primary school qualification, intermediate studies and university studies), presence of a computer at home (yes/no), knowledge of the existence of internet (yes/no), searches performed on urological conditions and influence of these consultations in their relationship with their doctor. The relationship between the use of internet and the different variables was evaluated using the Kruskall-Wallis test. A probability of the null hypothesis less than 0.05 was considered significant.

Results: A total of 1,111 questionnaires were received, of which 1,062 were useful for processing. The mean age was 60.98 with a standard error of 15.08. $18.4 \%$ were women. The population distribution by level of studies was: $22.2 \%$ uneducated, $43.5 \%$ with primary education qualification, $27.5 \%$ with intermediate educational level and $6.8 \%$ university graduates. $58.4 \%$ of patients denied having a computer at home, $37.7 \%$ do not know what internet is, $76.7 \%$ do not have an e-mail address and just $6.7 \%$ visit medical pages, although only $1.5 \%$ admit having asked their doctor about information received on internet. According to classification by age, patients under 30 have significantly greater knowledge of computers and internet ( $<0.001$ ). However, there were no significant differences shown between the age and the fact of asking about information received through internet ( $\mathrm{p}=0.1$ ). The most visited web pages were, in order of the most to least visited: tuotromedico.com, varicocele.com, aecc.es, wikipedia.com, prostatitis.org, ondasalud.com and mapfrecajasalud.com. The most searched for conditions were: chronic prostatitis (25\% of patients affected), testicular cancer ( $20 \%$ of patients), varicocele $(18.7 \%)$, Peyronie's disease or congenital penile curvature $(18.1 \%)$ and stenosis of the pyeloureteral junction (16.6 \%).

Conclusions: - The exploitation of the internet as an information tool on the part of patients is very low, due to the characteristics inherent to our population, such as the low level of studies. - Urological web pages should dedicate an extensive part to the most common conditions in the younger population groups, such as varicocele or prostatitis. However, it is logical to expect that these epidemiological patterns will modify with time. - The use of internet and computers in general should be promoted among the different population groups in the health area under study.

Keywords: Internet. Questionnaire. Epidemiology. 
$\mathrm{E}^{1}$ uso de internet ha crecido durante los últimos años. El número de individuos que han utilizado Internet alcanzó en el primer trimestre de 2006 los 16 millones, esto es un 47,9\% de la población entre 16 y 74 años. De ellos la inmensa mayoría, en torno al $86 \%$, han realizado un uso habitual el último mes. De diciembre de 2005 a junio de 2006 se ha producido un incremento cercano al medio millón de nuevos usuarios de Internet, lo que supone un incremeto de 2,5 puntos porcentuales. Además, en junio de 2006 el $39,1 \%$ de los hogares españoles estaban conectados a Internet, según los resultados de la encuesta sobre equipamiento y uso de tecnologías de la información y comunicación en los hogares publicados por el Instituto Nacional de Estadística ${ }^{1}$. El fácil acceso, la disponibilidad permanente y el anonimato de los usuarios, unido a la gran cantidad de páginas web dedicadas a la información de salud, ha permitido un mayor acceso a la información médica por parte de los profesionales y de los pacientes ${ }^{2}$.

Algunos estudios europeos muestran que hasta un $50 \%$ de los internautas han usado la red en alguna ocasión para obtener información relacionada con la salud. Esta cifra alcanza el 80 $\%$ entre los usuarios adultos de internet en Estados Unidos. En el año 2002, la búsqueda de información sobre salud, fue el tercer motivo de uso de internet, tras el correo electrónico y las ventas on-line ${ }^{3}$.

Nuestra hipótesis es que el aumento en los conocimientos de informática de la población general y la difusión de internet como herramienta de comunicación y conocimiento, permite a los pacientes tener un mayor entendimiento de sus patologías, por ello, se evaluará el uso de internet por parte de la población de un área sanitaria, así como el conocimiento extraído sobre sus patologías de carácter urológico.

\section{MATERIAL Y MÉTODOS}

En este estudio prospectivo se incluyeron todos los pacientes con edad superior a 18 años que acudieron a consulta hospitalaria de urología entre el 1 de Septiembre y el 31 de Diciembre de 2006, en un área sanitaria de 250,000 habitantes (Móstoles, Madrid). A todos los pacientes se les entregó antes de la consulta médica un cues- tionario autoadministrado para su cumplimentación (Fig. 1) sin limite de tiempo y no interviniendo el facultativo en su desarrollo en ningún caso. Por dicho motivo todos los pacientes que no fueron capaces de rellenar el cuestionario fueron excluidos. Las variables a estudio fueron la edad (menores de 30 años, entre 30 y 60 años y mayores de 60 años), el sexo, patología del paciente (únicamente se valorarán aquellas patologías con al menos 5 casos), el nivel educativo (ninguno, graduado escolar, estudios medios y estudios universitarios), presencia de ordenador en su casa (si/no), conocimiento de la existencia de internet $(\mathrm{si} / \mathrm{no})$, búsquedas realizadas sobre patologías urológicas e influencia de dichas consultas en su relación con el médico. Se evaluará la relación entre el uso de internet y las distintas variables mediante el test de Kruskall-Wallis. Se considerará significativa una verosimilitud de la hipótesis nula inferior a 0,05. Para el análisis estadístico se utilizará el software SPSS v13.0 (SPSS Inc, Chicago, Illinois, USA).

\section{RESULTADOS}

En total se recibieron 1111 cuestionarios de los cuales fueron útiles para su procesamiento 1062. La edad media fue de 60,98 años, con un

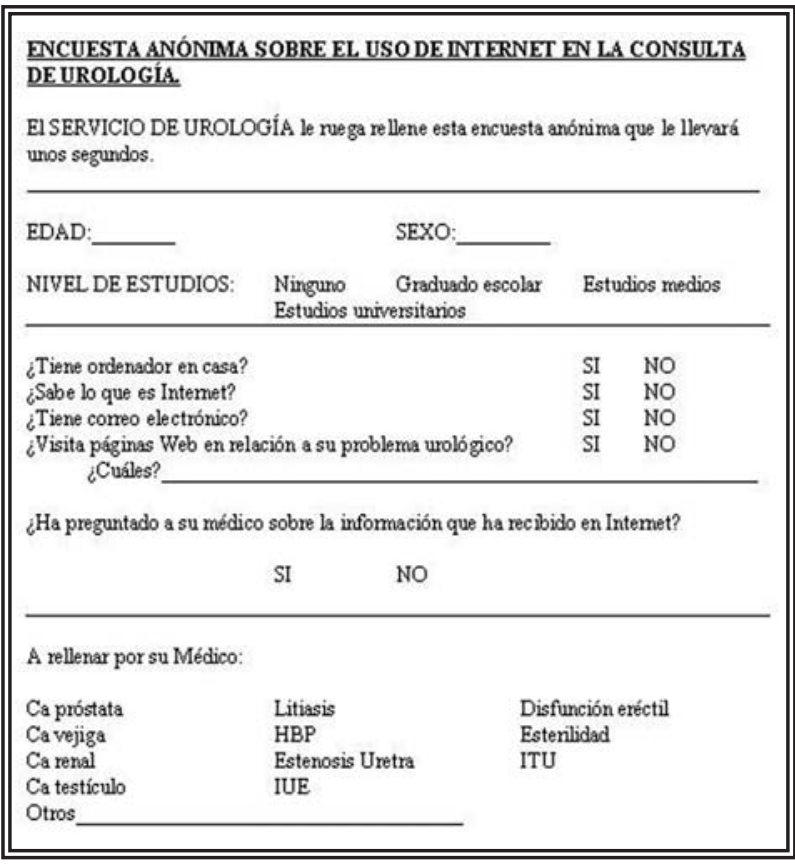

FIGURA 1. Cuestionario administrado a los pacientes para su cumplimiento. 
error estándar de 15,08. El 18,4 \% fueron mujeres. La distribución poblacional por nivel de estudios fue: $22,2 \%$ sin estudios, $43,5 \%$ con graduado escolar, 27,5 con un nivel educativo medio y un $6,8 \%$ de universitarios (Fig. 2). El 58,4\% de los pacientes niega tener ordenador en casa, el $37,7 \%$ desconoce lo que es internet, el $76,7 \%$ carece de dirección de correo electrónico y únicamente el 6,7\% visitan páginas médicas pero sólo el 1,5\% reconocen haber preguntado a su médico sobre la información recibida en internet (Fig. 3). Según la categorización por edades, los pacientes con edades inferiores a 30 años tienen significativamente unos mayores conocimientos en informática e internet $(\mathrm{p}<0,001)$. No obstante no se han evidenciado diferencias estadísticamente significativas entre la edad y el hecho de preguntar sobre la información recibida a través de internet $(\mathrm{p}=0,1)$.

Las páginas web más visitadas fueron por orden de más a menos visitadas: tuotromedico.com, varicocele.com, aecc.es, wikipedia.com, prostatitis.org, ondasalud.com y mapfrecajasalud.com. Ningún

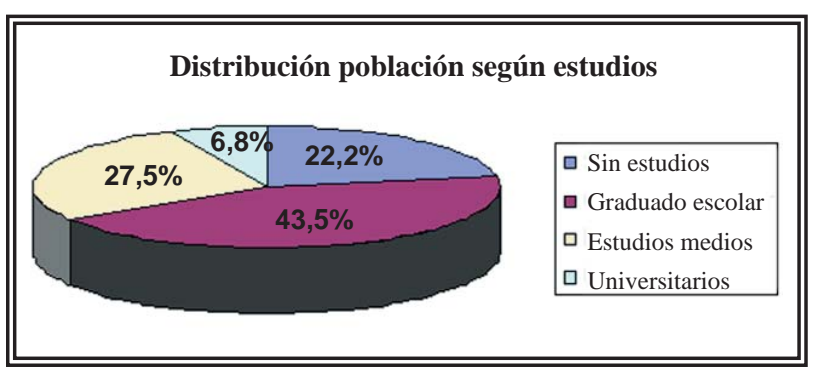

FIGURA 2. Distribución porcentual del nivel de estudios en el área sanitaria.

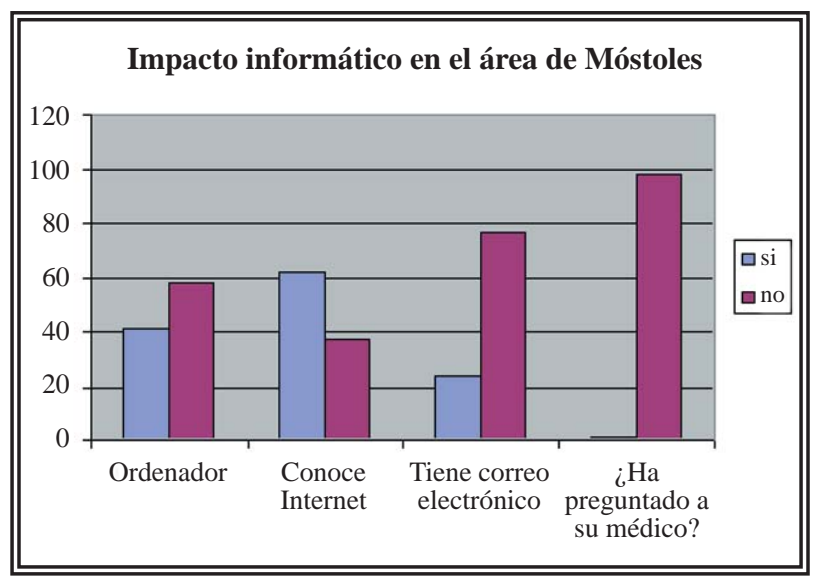

FIGURA 3. Distribución porcentual de las diferentes variables a estudio. paciente reconoció haber visitado la página web de la Asociación Española de Urología o de revistas españolas de ámbito urológico. Las patologías más buscadas fueron: prostatitis crónica $(25 \%$ de los pacientes afectos), cáncer de testículo (20 $\%$ de los pacientes), varicocele $(18,7 \%)$, enfermedad de Peyronie o incurvación congénita peneana $(18,1 \%)$ y estenosis de la unión pielo-uretereral (16,6\%).

Dentro de la patología oncológica únicamente 29 pacientes de 369 visitaron páginas web $(7,85$ $\%$, de las cuales si excluimos el cáncer de testículo, es el cáncer de próstata con un 8,3\% de los pacientes afectos la que más preocupa, seguida del cáncer vesical con un 7,59\%.

\section{DISCUSIÓN}

$\mathrm{El}$ interés por Internet como fuente de información por parte de los pacientes en otros campos de la medicina han mostrado que la búsqueda de información en la red de redes llega hasta el $58 \%$ y la búsqueda sobre patologías hasta un $17 \%$. En el caso de las enfermedades urológicas un estudio realizado por Hellawell en el año 2000 evidenció que el 19\% de los pacientes con patología urológica general y el $24 \%$ de los que sufrían procesos oncológicos buscaban información en Internet y que esa búsqueda de información se estaba incrementando progresivamente ${ }^{4}$. Sin embargo, nuestros resultados muestran que el uso de internet como fuente de información y conocimiento están muy por debajo de lo esperado puesto que menos del $7 \%$ de los encuestados han utilizado este medio para obtener más información. No obstante, la edad media de los pacientes fue de 61 frente a los 43 años del primer estudio.

En nuestro estudio se demuestra que los principales factores asociados al uso de internet son la edad así como el nivel educativo. Este hecho ha sido ampliamente contrastado por otros autores tal y como refiere Lorence en su estudio realizado en el año $2000^{5}$. En este estudio se relaciona el uso de Internet y el de la utilización de información en la red. De hecho los jóvenes son los que más acceden a Internet y los que menos utilizan la información sanitaria de la red. Schwartz halló también relación entre el acceso a Internet y el sexo, raza, e ingresos ${ }^{6}$. 
Cuando un paciente intenta encontrar información en la red, utiliza básicamente los datos obtenidos de los buscadores. No en todos los buscadores se encuentra la misma información ni de la misma calidad ante la misma pregunta. En un estudio realizado por Sacchetti se objetivó que la información obtenida por un buscador como Yahoo era mejor en contenido que la obtenida por otro buscador (HotBot). Sin embargo, al primero le faltaban páginas importantes que si aparecían en el segundo ${ }^{7}$.

A este hecho debemos añadir el que la información médica en internet tiene un grave problema, y es que no presenta ningún tipo de regularización, por lo que la información ofrecida puede ser no contrastada, de mala calidad o interesada, lo que puede generar conflictos en la relación médico-paciente. El impacto de internet ha sido evaluado por la Universidad Oberta de Catalunya que comprobó que el 4\% de los pacientes que habían realizado consultas médicas por internet, habían preguntado a su médico sobre dicha información ${ }^{8}$. En nuestro medio, únicamente el 1,5\% de los encuestados reconoció haber preguntado al médico, por lo que el uso de internet difícilmente influirá en la relación médico-paciente en nuestro medio en la actualidad.

Para incentivar la utilización de la información en Internet es importante el desarrollar una web en el que se tengan en cuenta las preferencias de los que las visitan, tal y como demostraron en Australia Pinnock y Jones. El impulso en la utilización de la web está en la mejora contínua en relación con la demanda de los pacientes tal y como demuestran en su trabajo los referidos Pinnock y Jones ${ }^{9}$.

Hay un hecho relevante referido en la bibliografía y es que muchos de los pacientes que utilizan la red para obtener la información luego preguntan a sus médicos acerca de lo que han encontrado. En otras ocasiones son los propios médicos los que remiten a los pacientes a estas webs para que dispongan de más información. Es labor del médico el dirigir a los pacientes a lugares en donde la información se actualice y sea fia$b^{6}{ }^{6}$. Las sociedades urológicas deben responsabilizarse de los sitios web y de la información allî expuesta incluyendo medicina basada en la evi- dencia, ofreciendo asistencia a aquellos pacientes que quieran comparar la opinión de sus especialistas con aquella obtenible en Internet ${ }^{7,10}$.

El estudio tiene varias limitaciones. Por un lado los resultados se extraen de una consulta hospitalaria de urología, por lo que no es directamente extrapolable a la población general. De hecho según el Instituto Nacional de Estadística de España, el 62 \% de la población tiene estudios secundarios o universitarios, frente al $35 \%$ del área sanitaria a estudio ${ }^{11}$.

Otra limitación importante es que no se han valorado el nivel científico de las páginas webs consultadas, ni la interpretación recibida por los pacientes ni trasmitida a los médicos.

\section{CONCLUSIONES}

1. La explotación de internet como herramienta de información por parte de los pacientes es muy baja, debido a las características inherentes de la población, como son el bajo nivel de estudios.

2. Las páginas web urológicas deberían dedicar una parte amplia para la patología más frecuente en los grupos de población más jóvenes, como el varicocele o la prostatitis. No obstante, es lógico esperar que estos patrones epidemiológios se modifiquen con el paso del tiempo.

3. Se debería fomentar el uso de internet y de la informática en general entre los distintos grupos poblacionales en el área sanitaria a estudio.

\section{REFERENCIAS}

1. Ministerio de Industria, Turismo y Comercio. Informe: Encuesta sobre equipamiento y uso de Tecnologias de Información y Comunicación en las viviendas (4 Octubre 2006). http://observatorio.red.es/indicadores/areas/ciudadanos/internet/individuos_internet.html. Última entrada $4 / 2 / 2007$.

2. Alarcón O, Baudet JS, Sánchez Del Río A, Dorta MC, De La Torre M, Socas MR, et al. Uso médico de internet entre los pacientes de una consulta general de enfermedades del aparato digestivo. Gastroenterol Hepatol. 2006;29(5):286-290.

2. Alarcón O, Baudet JS, Sánchez Del Río A, Dorta MC, De La Torre M, Socas MR, et al. Internet use to obtain health information among patients attending a digestive diseases office. Gastroenterol Hepatol. 2006;29(5):286-290.

3. En Pew Internet/American Life Project. http://www.pewinternet.org/pdfs/PIP_Online_Health_2006.pdf. Ültima entrada $4 / 2 / 2007$.

4. Hellawell GO, Turner KJ, Le Monnier KJ, Brewster SF. Urology and the Internet: an evaluation of internet use by urology patients and of information available on urological topics. BJU.Int. 200;86(3):191-194. 
5. Lorence DP, Park H. New technology and old habits: The role of age as a technology chasm. Technol Health Care. 2006; 14(2):91-96.

6. Schwartz KL, Roe T, Northrup J, Meza J, Seifeldin R, Neale AV. Family medicine patients' use of the Internet for health information: a MetroNet study. J Am Board Fam Med. 2006; 19(1):39-45.

7. Sacchetti P, Zvara P, Plante MK. The Internet and patient education resources and their reliability: focus on a select urologic topic. Urology. 199;53(6):1117-1120.

8. Vivas P, Armayones M, Boixadós M et al. Salud e internet: búsqueda temática y calidad de su contenido. http://www. ouc.edu/in3/dt/20375/index.htm

9. Pinnock CB, Jones C; Education Committee of the Australian Prostate Cancer Collaboration. Meeting the information needs of Australian men with prostate cancer by way of the internet. Urology. 2003;21(3):1198-1203.

10. Sole Balcells FJ. The Internet in medicine. A help, a problem, a cause of errors?. Actas Urol.Esp. 2003;27(3):180184.

11. Instituto Nacional de Estadística de España. www.ine.es/ inebase. Última entrada 4/2/2007.

Correspondencia autor: Dr. D. Santos Arrontes.

Servicio de Urología. Hospital de Móstoles.

Rio Júcar, s/n - 28935 Móstoles (Madrid). Tel: 916648754

E-mail autor: dsantosa@gmail.com

Información artículo: Original - Nuevas tecnologías y urología

Trabajo recibido: junio 2007

Trabajo aceptado: septiembre 2007 